\title{
Political Risk in Hong Kong and Taiwan: Pricing the China Factor
}

\author{
Ephraim Clark \\ Ceram School of Management
}

\begin{abstract}
This paper measures how the risk associated with foreign direct investment in the prosperous, liberal economies of Hong Kong and Taiwan is affected by the prospect of reunification with the poor, politically and economically backward mainland. This China factor is modeled as a jump to a higher political risk level. I find that the China factor effect is substantial on Taiwan but still almost six times higher on Hong Kong. Nevertheless, Hong Kong's political risk is considerably lower than China's, thereby confirming the intuition that Hong Kong could be a lower risk back door avenue to the potentially lucrative Chinese market. (JEL Classification: G31)
\end{abstract}

\section{Introduction}

Hong Kong is currently a British colony of 6 million inhabitants $98 \%$ of whom are ethnic Chinese. Taiwan is an island of 21 million inhabitants, 99\% of whom are ethnic Chinese. Both are considered Chinese provinces and both are major economic success stories in their own right. In 1994 Hong

* Correspondence Address: Ephraim Clark, CERAM School of Management, Sophia Antipolis, 06902 FRANCE. (Tel) +33-1-47-57-72-34, (Fax) +33-4-93-65-45-24, (E-mail) clark@ceram.fr; Thanks to my discussant at the conference, Hamid Beladi, and an anonymous referee for helpful comments.

(C)1998 - Institute for International Economics, Sejong Institution. All rights reserved. 
Kong had a per capita GDP of $\$ 23,000$. Between 1980 and 1995 its growth rate averaged over 6\% per year. In 1994 Taiwan had a per capita GDP of $\$ 13,000$ and its annual growth rate between 1980 and 1995 was close to $8 \%$. Both are characterized by economic liberalism and minimal state intervention. ${ }^{1}$ Hong Kong, however, is set to revert to mainland control on July 1, 1997 and Taiwan claims it seeks eventual reunification with the mainland, in spite of rumors of independence and the democratic presidential election of March 1996. In both cases, the prospect of reunification with the mainland darkens the sky with clouds of uncertainty for foreign companies with an ongoing activity in one of the two provinces or contemplating an eventual presence there. The problem is mainland China. Notwithstanding an annual growth rate of almost 10\% between 1980 and 1995, mainland China is poor its per capita GDP was only $\$ 2,500$ in 1994 . More importantly, it clings doggedly to the discredited Marxist economic and political ideology featured by state planning and government intervention. Thus, foreign firms with an ongoing or projected presence in Hong Kong or Taiwan are faced with analyzing the consequences of an eventual merger with a regime that is so idealogically, politically and economically different. To this end they must attempt to come up with adequate answers to a number of thorny questions: 1) What is the likelihood of reunification actually occurring; 2) What is the likelihood that reunification will cause substantial changes in the economic and political environment; 3) If substantial change does occur, what form will it take and what are the consequences for corporate operations.

The question addressed in this paper is how the risk associated with foreign direct investment (FDI) in the prosperous, liberal economies of Hong Kong and Taiwan is affected by the prospect of reunification with the poor, politically and economically backward mainland. More specifically, I develop a model for pricing political risk in Hong Kong and Taiwan where the reunification factor figures explicitly. Using standard techniques in stochastic calculus, I model political risk as an index of exposure to loss. I adopt the very

1. A recent study of 102 countries from 1975 to 1995 finds Hong Kong 1st and Taiwan 16th in overall economic freedom. See: James Gwartney, Robert Lawson, and Walter Block, Economic Freedom of the World: 1975-1995, 〈Vancouver: Fraser Institute [1996]>. See Kobrin [1979] and Roddock [1986] for a discussion the issues involves in political risk. 
broad definition of political risk as the probability of politically motivated change that affects the outcome of foreign direct investment and make a distinction between explicit events and ongoing change. Explicit events take the form of legislation or decrees such as price controls, taxes, devaluations, etc. The nature of explicit events is that they arrive intermittently at discrete intervals and that they generate an actual loss. Explicit events can be represented by a Poisson jump process. Ongoing change takes the form of continuous activity such as macroeconomic management and monetary policy, legislation, or social and political evolution that affects some or all aspects of the FDI's overall environment. Thus, in my model, ongoing change impacts on the level of what can be lost in the case of an explicit event and can be represented by geometric Brownian motion. As an example of the distinction between explicit events and ongoing change, take the case of an overexpansive monetary policy with a fixed exchange rate. The over-expansive monetary policy generates the ongoing changes that affect what will be lost if the explicit event of a devaluation takes place. On the other hand, with a floating exchange rate, changes in the value of the host country's currency would be considered ongoing change and not an explicit event. With these distinctions in mind, the effects of political risk on the outcome of a foreign direct investment can be measured as the value of an insurance policy that reimburses all losses resulting from the political event or events in question.

Not surprisingly, due to the imminence of Hong Kong's reunification, I find a stronger China factor effect on Hong Kong than on Taiwan. What is surprising, however, is the degree of difference between the two. For Hong Kong, the China factor raises the political risk level by $329 \%$ in the worst case scenario and by $149 \%$ in the best case. For Taiwan the figures are $60 \%$ and $24 \%$ respectively. Interestingly, I show that even in the worst case scenario, an FDI in Hong Kong is considerably less risky than an equivalent investment in China. This confirms the intuition that Hong Kong could be a lower risk back door avenue to the potentially lucrative Chinese market. I also show that otherwise acceptable projects become unacceptable when the China factor is taken into consideration. Besides the insights on political risk specific to Hong Kong and Taiwan due to the China factor, this paper contributes to the political risk literature in general insofar as it develops for the first time (to my knowledge) a rigorous methodology for measuring the 
impact on FDI of the combined effects of a randomly fluctuating exposure to loss with potential random changes in country risk levels.

The paper is organized as follows. Section II uses standard techniques of stochastic calculus to develop a model for measuring the cost of political risk when the level of exposure varies randomly with time and the arrival rate of losses is also random but subject to change that also arrives at a random rate. Section III analyzes the model's general characteristics and estimates the parameters for China, Hong Kong and Taiwan. Section IV applies the model to estimate the cost of political risk for China, Hong Kong and Taiwan, compares the situations of the three with and without the China factor, and then shows how the information can be incorporated into the capital budgeting process. Section $\mathrm{V}$ presents my concluding remarks.

\section{Modeling Changing Levels of Political Risk}

Let $x(t)$ represent the level of loss in the case of political intervention where $x$ evolves according to a process of geometric Brownian motion:

$$
d x(t)=\alpha x(t) d t+\sigma x(t) d z(t)
$$

where $\alpha=$ the rate of growth of exposure to loss with $\alpha<0 ; \alpha>0$; $\alpha=0$. It depends on the rate of growth of the value of the investment determined by the investment's internal rate of return multiplied by the rate of reinvestment out of profits.

$$
\begin{aligned}
& d z(t)=\text { a Wiener process with zero mean and variance equal to } d t \\
& \sigma^{2}=\text { the variance of } d x / x
\end{aligned}
$$

Suppose that loss causing political events occur at random times according to a Poisson arrival process where $q_{1}(t)$ is a random variable that increases by steps of 1 every time a Poisson event occurs and $\lambda$ is a constant intensity parameter such that:

$$
d q_{1}(t)=\left\{\begin{array}{llr}
1 & \text { with probability } \quad \lambda d t \\
0 & \text { with probability } 1-\lambda d t
\end{array}\right.
$$

This means that in the absence of the China factor, losses arrive at a rate of $\lambda d t$ and that $\lambda$ is the current political risk probability parameter. Thus, in the absence of effects associated with possible reunification, the expected loss 
per $d t$ is equal to $\lambda x(t) d t$.

Now suppose that because of reunification there is a chance that the country will become riskier (less risky). The loss level is the same but losses occur more frequently (less frequently) according to a Poisson process $q_{2}(t)$ where

$$
d q_{2}(t)= \begin{cases}1 & \text { with probability } \phi d t \\ 0 & \text { with probability } 1-\phi d t\end{cases}
$$

The expected loss per $d t$ increases from $\lambda x(t) d t$ to $\phi x(t) d t$ where $\phi$ is the political risk probability parameter prevailing after reunification.

It is generally accepted that mainland China has much to gain from the ongoing organization and management of Hong Kong and Taiwan and much to lose if it upsets the applecart. Consequently, there is no certainty that the reunification itself will trigger a change to the higher risk level. Suppose that the change from one risk level to another, if it comes, is governed by a Poisson process $q_{3}$ with intensity parameter $\rho$ where

$$
d q_{3}(t)= \begin{cases}1 & \text { with probability } \quad \rho d t \\ 0 & \text { with probability } 1-\rho d t\end{cases}
$$

Thus, $\rho$ represents the probability that the mainland will actually achieve reunification and if it does that it will pursue a policy of increased intervention in the economies of the reunified provinces.

To evaluate the cost of both levels of political risk and the probability that the second level will actually be realized, we start by evaluating the cost of the second risk level. Let $V$ represent the value of an insurance policy covering the investment against losses arising from the second level of political risk. Because of the perpetual nature of a direct investment, $V$ is a perpetual claim and, consequently, its value does not depend on time. Thus, $V$ is a function of the exposure to losses arising from the second level of political risk and the probability of an explicit political event actually occurring:

$V=V\left(x(t), q_{2}(t)\right)=$ value of the insurance policy covering the investment against losses arising from the second level of political risk.

Using Ito's Lemma and taking expectations, gives:

$$
E(d V)=V^{\prime}(x(t)) \alpha x(t) d t+\frac{1}{2} V^{\prime \prime}(x(t)) \sigma^{2} x(t)^{2} d t
$$


and the total return on the insurance policy is equal to $E(d V)+\phi x(t) d t$. Assume that the world economy is risk neutral and that the risk free interest rate is constant at $r^{2}$ The insurance policy will then yield the riskless rate so that $E(d V)+\phi x(t) d t=r V(x(t)) d t$. Substituting from (2) and simplifying, gives the following differential equation:

$$
\frac{1}{2} \sigma^{2} x(t)^{2} V^{\prime \prime}(x(t))+V^{\prime}(x(t)) \alpha x(t)-r V(x(t))+\phi x(t)=0
$$

Under the assumption that $r>\alpha$, the general solution to (3) is:

$$
V(x(t))=\frac{\phi x(t)}{r-\alpha}+A_{1} x(t)^{\gamma_{1}}+A_{2} x(t)^{\gamma_{2}}
$$

where $\gamma_{1}>1$ (because $r>\alpha$ ) and $\gamma_{2}<0$ are the roots to the quadratic equation in $\gamma$ :

$$
\begin{aligned}
& \frac{\sigma^{2}}{2} \gamma^{2}+\left(\alpha-\frac{\sigma^{2}}{2}\right) \gamma-r=0 \\
& \gamma_{1}, \gamma_{2}=\frac{\left(\alpha-\sigma^{2} / 2\right) \pm \sqrt{\left(\alpha-\sigma^{2} / 2\right)^{2}+2 \sigma^{2} r}}{\sigma^{2}}
\end{aligned}
$$

An increase in the value of the insurance policy due to a small increase in the value of exposure should always be finite, which rules out speculative bubbles. Furthermore, when the level of exposure is equal to zero, the insurance policy is worthless. Thus, the boundary conditions are:

$$
\begin{aligned}
& V^{\prime}(\infty)<\infty \\
& V(0)=0
\end{aligned}
$$

Since $\gamma_{1}>1$, the first boundary condition implies that $A_{1}=0$. The second boundary condition implies that $A_{2}=0$. Thus, the solution is

2. An alternative procedure involves defining a spanning asset for $x$ and using it in an asset pricing model to determine $x$ 's required rate of return. A riskless portfolio consisting of one unit of $V(x(t))$ and a short position of $-V^{\prime}(x(t))$ units of $x$ is then constructed and Ito's lemma applied to give the same differential equation as (3) with $\alpha^{*}$ replacing $\alpha . \alpha^{*}$ represents the growth rate adjusted for discounting at the riskless rate of interest. Using the CAPM, for example, $\alpha^{*}=\alpha-\beta\left(\bar{r}_{m}-r\right)$ where $\bar{r}_{m}$ is the expected return on the market and $\beta$ is the covariance of the return on the market with the return on the insurance policy divided by the variance of the return on the market. 


$$
V(x(t))=\frac{\phi x(t)}{r-\alpha}
$$

The value of the insurance policy is equal to the present value of expected losses discounted at the riskless rate.

Let $W$ represent the value of an insurance policy that includes both levels of risk:

$$
W=W\left(x(t), q_{1}(t), q_{2}(t), q_{3}(t)\right) .
$$

Using Ito's Lemma and taking expectations, gives:

$$
E(d W)=W^{\prime}(x(t)) \alpha x(t) d t+\frac{1}{2} W^{\prime \prime}(x(t)) \sigma^{2} x(t)^{2} d t
$$

Expected cash flows arising from losses are equal to $\lambda x(t) d t$, the instantaneous cash flow arising from the first level of risk, plus $\rho\left(\frac{\phi x(t)}{r-\alpha}-W(x(t))\right) d t$, the value of the expected net cash flows arising from the change in risk levels. In other words, if the change in risk levels occurs, the insuree gives up $W(x(t))$ to gain $\frac{\phi x(t)}{r-\alpha}$ with a probability of $\rho d t$. Assuming, as before, that the world economy is risk neutral and that the risk free interest rate is constant at $r$, the insurance policy will then yield the riskless rate so that

$$
E(d W)+\lambda x(t) d t+\rho\left(\frac{\phi x(t)}{r-\alpha}-W(x(t))\right) d t=r W(x(t)) d t .
$$

Substituting from (8) and simplifying, gives the following differential equation:

$$
\begin{gathered}
\frac{1}{2} W^{\prime \prime}(x(t)) \sigma^{2} x(t)^{2}+W^{\prime}(x(t)) \alpha x(t)-r W(x(t) \\
+\lambda x(t)+\rho\left(\frac{\phi x(t)}{r-\alpha}-W(x(t))\right)=0
\end{gathered}
$$

The general solution to this equation is:

$$
W(x(t))=\left[\frac{\phi \rho}{(r-\alpha)(r+\rho-\alpha)}+\frac{\lambda}{(r+\rho-\alpha)}\right] x(t)+B_{1} x(t)^{\gamma_{1}}+B_{2} x(t)^{\gamma_{2}}
$$

As before the boundary conditions are:

$$
\begin{aligned}
& W^{\prime}(\infty)<+\infty \\
& W(0)=0
\end{aligned}
$$


(11) implies that $B_{1}=0$ and (12) implies that $B_{2}=0$. Thus:

$$
W(x(t))=\left\lceil\frac{\phi \rho}{(r-\alpha)(r+\rho-\alpha)}+\frac{\lambda}{(r+\rho-\alpha)}\right\rceil x(t)
$$

The value of the insurance policy covering the political risk on an investment in a low (high) risk country that might become a high (low) risk country is equal to the present value of expected cash flows resulting from losses discounted at the riskless rate increased by the probability that the change in riskiness will actually occur. The effect of the China factor is reflected in $\frac{\phi \rho}{(r-\alpha)(r+\rho-\alpha)} x(t)$.

\section{Characteristics of the Solution}

\section{A. General Features}

By taking the first partial derivative with respect to each of the parameters in (13), we can get a feeling for how the model is likely to behave in practice. Table 1 summarizes the results. The cost of political risk increases with the mean arrival rate of explicit political events $(\lambda$ and $\phi)$ and the rate of growth of the exposure to risk $(\alpha)$. It decreases with the interest rate $(r)$. If the second level of political risk is greater than the current level $(\phi>\lambda)$, an increase in the probability of change from one level of risk to another $(\rho)$ increases the cost of political risk. In the opposite case, it decreases it.

It is also important to know the length of time that is likely to elapse before the change from one risk level to another. In the interval $[0, T]$, the probability that no change occurs is $e^{-\rho T}$. The probability that change occurs in the short interval $[T, T+d T]$ is $e^{-\rho T} \rho d T$. Therefore, the expected time before the change occurs is

Table 1

The Effect of Individual Parameters on the Cost of Political Risk

\begin{tabular}{|c|c|c|c|c|c|}
\hline$\frac{\partial \mathrm{W}}{\partial \phi}$ & $\frac{\partial \mathrm{W}}{\partial \lambda}$ & $\frac{\partial \mathrm{W}}{\partial \alpha}$ & $\frac{\partial \mathrm{W}}{\partial r}$ & $\frac{\partial \mathrm{W}}{\partial \rho}$ & $\frac{\partial \mathrm{W}}{\partial \rho}$ \\
\hline positive & positive & positive & negative & $\begin{array}{c}\text { positive if } \\
\phi>\lambda\end{array}$ & $\begin{array}{c}\text { negative if } \\
\phi<\lambda\end{array}$ \\
\hline
\end{tabular}




$$
E[T]=\int_{0}^{\infty} T \rho e^{-\rho T} d T=\frac{1}{\rho}
$$

Thus, if we know $\rho$, we know the average length of time before the political risk level changes. Knowing the expected duration of the status quo can be important for strategic planning and capital budgeting. ${ }^{3}$

\section{B. Parameter Estimation}

Applying the model to value the cost of political risk requires estimating the parameters $r, \alpha, \lambda, \phi, \rho$. For the riskless rate, I use the average yield to maturity on a long term US government bond between 1985 and 1994 so that $r=8 \%$. In order to concentrate on the political risk parameters, $\lambda, \phi$, and $\rho$, rather than on a particular type of investment, I let $\alpha=0 .{ }^{4}$

To estimate $\lambda$, I refer to the freedom index of Gwartney, Lawson, and Block [1996]. This index, the fruit of a process begun in 1984 with contributions from 61 people and the participation of eleven research institutes, attempts to measure the extent to which rightly acquired property is protected and individuals are free to engage in voluntary transactions. ${ }^{5}$ It is based on 17 objective components that are allocated into four major areas: (1) money and inflation, (2) government operations and regulations, (3) takings and discriminatory taxation, and (4) international exchange. The 17 components are relevant to the definition of explicit political risk outlined above. Thus, since the index reflects relative levels of economic freedom, I

3. Take, for example, the case where the investment would be unprofitable at the higher level of risk. The expected time before a change in the risk level could be compared with the expected time for the investment to generate an NPV equal to zero. A zero NPV time frame less than the expected time to a change in the risk level would be a strong argument for undertaking the investment since it is likely that in this case the investment will at least break even.

4. Choosing $\alpha$ is equivalent to choosing the growth rate of the FDI since $\alpha$ depends on the rate of growth of the FDI. $\alpha=0$ implies that the FDI has a constant net cash flow and that there is no net investment.

5. The Gwartney et al. index is the most complete and comprehensive of its kind, reflecting fundamental research that attempts to deal with the key methodological issues involved in the creation of such an index. The Heritage Foundation Index of Economic Freedom, for example, reflects much of the work done by the Fraser Institute but is less comprehensive. 
take $\lambda$ as proportional to the reciprocal of the index. On a scale of 1 to 10 their index is 9.1 for Hong Kong and 6.6 for Taiwan. Thus:

$$
\begin{aligned}
& \lambda=\kappa 1 / 9.1 \cong \kappa 0.11 \text { for Hong Kong and } \\
& \lambda=\kappa 1 / 6.6 \cong \kappa 0.15 \text { for Taiwan. }
\end{aligned}
$$

To estimate $\phi$, China's political risk parameter, I assume that China's economic freedom parameter is at least as low as Hungary's 3.3 and no lower than Zaire's 1.9. Again supposing that the political risk parameter is proportional to the reciprocal of the freedom index, $\phi$ will lie between:

$$
\phi=\kappa 1 / 3.3 \cong \kappa 0.30
$$

and

$$
\phi=\kappa 1 / 1.9 \cong \kappa 0.53
$$

To estimate $\rho$, the probability that the mainland will actually achieve reunification and if it does, that it will pursue a policy of increased intervention in the economies of the reunified provinces, I refer to figures supplied by Credit Risk International, a country risk rating agency based in Paris. To assess each criterion, Credit Risk International followed the "Delphi technique". This methodology involves submitting a series of identical questions to a group of experts. The experts, who remain anonymous to each other, receive continual feedback on the responses of the other members of the group. The goal of the exercise is to reach a group position on a particular issue. From this it is estimated that there is a $50-50$ chance that China will actually pursue and effectively implement a policy of increased intervention. For Hong Kong it is estimated that there is a 99\% chance of reunification and for Taiwan a $5 \%$ chance of reunification. ${ }^{6}$ Thus, assuming linear indepen-

6. Given the reunification timetable for Hong Kong, the probability at the end of 1995 was given as "at least 99\%" and was expected to rise toward $100 \%$ if reunification proceeded on schedule. However, the elasticity of the value of the insurance policy with respect to $\rho$ is very low $(0.084$ at $\rho=0.495, \phi=0.3$, and $\kappa=1$, for example). Therefore, the forecast evolution of the reunification parameter has only a marginal effect (less than 0.1 of $1 \%$ ) on the value of the insurance policy. For Taiwan the elasticity of the value of the insurance policy with respect to $\rho$ is also very low: 0.147 for $\rho=$ $0.025, \phi=0.3$, and $\kappa=1$. 
dence between the probability of reunification and the probability of pursuing and implementing a policy of increased intervention:

$$
\rho=0.5 \times 0.99=0.495 \text { for Hong Kong }
$$

and

$$
\rho=0.5 \times 0.05=0.025 \text { for Taiwan. }
$$

Thus the expected time to a change in the level of political risk is $1 / \rho=2.02$ years for Hong Kong and $1 / \rho=40$ years for Taiwan. Table 2 summarizes the parameters.

Table 2

Parameter Estimates

\begin{tabular}{|l|c|c|c|c|c|c|c|c|}
\hline & $\mathbf{r}$ & $\alpha$ & $\lambda$ & $\phi$ high & $\phi$ low & $\phi$ med. & $\rho$ & $\mathbf{1} / \rho$ \\
\hline HK & 0.08 & 0 & $\kappa 0.11$ & - & - & - & 0.495 & $2.02 \mathrm{yrs}$ \\
\hline Taiwan & 0.08 & 0 & $\kappa 0.15$ & - & - & - & 0.025 & $40.00 \mathrm{yrs}$ \\
\hline China & 0.08 & 0 & - & $\kappa 0.53$ & $\kappa 0.30$ & $\kappa 0.385$ & - & - \\
\hline
\end{tabular}

\section{Pricing the Cost of Political Risk for FDI Decisions}

Using the parameters in Table 2 with $x(0)=\$ 1$, Table 3 shows the cost of political risk to Hong Kong and Taiwan for different levels of $\kappa$ in the absence of the China factor and Table 4 shows the cost of political risk to China itself. In the absence of the China factor, the cost of political risk is about 36\% higher for Taiwan than for Hong Kong. China's cost of political risk is about 4.8 times higher than Hong Kong's and 3.5 times higher than Taiwan's for the highest level of $\phi$. For the lowest level of $\phi$ it is 2.73 times and 2 times higher respectively.

Table 3

The Cost of Political Risk for Hong Kong, Taiwan in the Absence of the China Factor

\begin{tabular}{|l|c|c|c|}
\hline & $\kappa=1$ & $\kappa=0.75$ & $\kappa=0.5$ \\
\hline Hong Kong & 1.3750 & 1.0313 & 0.6875 \\
\hline Taiwan & 1.8750 & 1.4062 & 0.9375 \\
\hline
\end{tabular}


Table 4

The Cost of China's Political Risk

\begin{tabular}{|l|c|c|c|}
\hline & $\kappa=1$ & $\kappa=0.75$ & $\kappa=0.5$ \\
\hline China $(\phi$ high $)$ & 6.5863 & 4.9397 & 3.2931 \\
\hline China $(\phi$ low $)$ & 3.7538 & 2.8153 & 1.8769 \\
\hline China $(\phi$ medium $)$ & 4.8125 & 3.6094 & 2.4063 \\
\hline
\end{tabular}

When the China factor is accounted for, the picture changes considerably. From Table 5 we can see that the China factor increases Hong Kong's cost of political risk by $149 \%$ in the best case scenario and by $329 \%$ in the worst case scenario. Nevertheless, as can be seen by comparing these results with those in Table 4, Hong Kong's cost of political risk is still considerably lower than that of China. Thus, given the growing economic and political links between the two and the proximity of the reunification date, an investment in Hong Kong could be a strategy for a relatively low risk path to the potentially lucrative Chinese market. In contrast to Hong Kong, the China factor increases Taiwan's political risk by only $60 \%$ in the worst case and $24 \%$ in the

\section{Table 5}

\section{Cost of Political Risk for Hong Kong and Taiwan when the China Factor is Present}

\begin{tabular}{|c|c|c|c|}
\hline Hong Kong & $\phi$ high & $\phi$ low & $\phi$ medium \\
\hline$\kappa=1.00$ & 5.8946 & 3.4196 & 4.3342 \\
\hline$\kappa=0.75$ & 4.4209 & 2.5647 & 3.2507 \\
\hline $\begin{array}{l}\kappa=0.50 \\
\text { \% change compared to } \\
\text { absence of China Factor }\end{array}$ & 2.9473 & 1.7100 & 2.1671 \\
\hline Taiwan & $329 \%$ & $149 \%$ & $215 \%$ \\
\hline $\begin{array}{l}\kappa=1.00 \\
\kappa=0.75\end{array}$ & 3.0060 & 2.2314 & 2.5744 \\
\hline $\begin{array}{l}\kappa=0.50 \\
\% \text { change compared to } \\
\text { absence of China Factor }\end{array}$ & 2.2545 & 1.7411 & 1.9308 \\
\hline
\end{tabular}


best case. Some examples will illustrate how the foregoing information can be used in the capital budgeting process.

To incorporate the cost of political risk into the capital budgeting process, I consider the overall investment as the value of a portfolio composed of the value of the project in the absence of political risk plus the value of the short position in the insurance policy. Finding the value of the portfolio involves estimating the project's net present value (NPV) or adjusted net present value (APV) as if there were no political risk and then subtracting the cost of political risk as estimated in the model. ${ }^{7}$

Consider a permanent investment of US\$10 million. No new investment is anticipated. Consequently, the expected rate of growth of net cash flows is zero and all profits are scheduled to be repatriated. In the absence of specific political risk, the certainty equivalent net cash flow is estimated at US\$1 million. At the riskless rate of $8 \%$ the investment is worth $\$ 12.5$ million $\left(\int_{0}^{\infty} \$ 1 e^{-0.08 t} d t=\$ 12.5\right)$ and its NPV is $\$ 2.5$ million $(\$ 12.5-\$ 10)$. The exposure to political risk at the outset is estimated to be the full value of the net cash flow of $\$ 1$ million $(x(0)=\$ 1$ million). Since the cash flow is not expected to grow, $\alpha=0$, the NPV of the overall portfolio is equal to the NPV of the FDI (\$2.5 million) minus the value of the insurance policy. Using the parameters of Table 2, Table 6 shows the results of this exercise for an FDI in Hong Kong and Taiwan with and without the China factor for different values of $\kappa$ and $\phi$. Without the China factor, the FDI has a positive NPV for both Hong Kong and Taiwan for all levels of $\kappa$, although it is considerably higher in Hong Kong. When the China factor is considered, the situation is completely different for Hong Kong. The NPV is positive only for the low $\phi$ and medium values of at the lowest value of $\kappa$. Furthermore, as we can see

7. Because of the risk neutrality assumed in the model, proceeding in this way either implies the assumption of linear independence between all political risk and the market portfolio or requires working with the investment's certainty equivalent cash flow. If the alternative evaluation procedure outlined in footnote 2 is used, these constraints no longer hold. The obvious difficulty with the procedure in footnote 2 is finding an appropriate spanning asset. In some situations, however, this difficulty can be overcome. Clark [1991], for example, has proposed a methodology for estimating systematic country specific economic risk. See Shapiro [1978] and Stonehill and Nathanson [1968] for the issues involved in international capital budgeting. 
Table 6

Net Present Value of Investment 1 with and without the China Factor

\begin{tabular}{|l|c|c|r|r|r|}
\hline \multicolumn{2}{|c|}{} & without China & \multicolumn{1}{c|}{$\phi$ high } & \multicolumn{1}{c|}{$\phi$ low } & $\phi$ medium \\
\hline \multirow{4}{*}{ Hong Kong } & $\kappa=1.00$ & 1.1250 & -3.3946 & -0.9196 & -1.8342 \\
\cline { 2 - 6 } & $\kappa=0.75$ & 1.4687 & -1.9209 & -0.0647 & -0.7507 \\
\cline { 2 - 6 } & $\kappa=0.50$ & 1.8125 & -0.4473 & 0.7900 & 0.3329 \\
\hline \multirow{3}{*}{ Taiwan } & $\kappa=1.00$ & 0.6250 & -0.5060 & 0.2686 & -0.0744 \\
\cline { 2 - 6 } & $\kappa=0.75$ & 1.0938 & 0.2455 & 0.7589 & 0.5692 \\
\cline { 2 - 6 } & $\kappa=0.50$ & 1.5625 & 0.9970 & 1.3393 & 1.2128 \\
\hline
\end{tabular}

Table 7

Expected Time to Zero NPV

\begin{tabular}{|l|c|c|c|}
\hline & $\kappa=1.00$ & $\kappa=0.75$ & $\kappa=0.50$ \\
\hline Hong Kong & 28.64 & 25.69 & 23.43 \\
\hline Taiwan & 35.42 & 28.96 & 25.02 \\
\hline
\end{tabular}

in Table 7, the expected time to zero NPV with the current level of political risk is far longer than the expected time to the expected change in the political risk level of 2.02 years. $^{8}$ Thus, even if the investment is profitable at the lower risk level it is unlikely that the change to the higher risk level will come late enough to allow the investment to become profitable. For Taiwan, on the other hand, the NPV is positive in all but two cases: the high and medium values of $\phi$ for the highest value of $\kappa$. The expected time to zero NPV is also far lower than the expected time to the change in the political risk level of 40 years. Consequently, even in the cases where the NPV of the portfolio is negative, there is a good chance that the investment will pay off before the unfavorable event hits.

8. This is calculated as the value of $T$ that equates the value of the cost of the investment plus the cost of the current level of political risk with the value of the investment:

$$
\$ 10+\int_{0}^{T} \$ 1 \kappa \lambda e^{-r t} d t=\int_{0}^{T} \$ 1 e^{-r t} d t
$$




\section{Conclusion}

This paper addresses the problem of how the risk associated with foreign direct investment (FDI) in the prosperous, liberal economies of Hong Kong and Taiwan is affected by the prospect of reunification with the poor, politically and economically backward mainland. Using standard techniques in stochastic calculus, I model the cost of political risk as the value of an insurance policy that reimburses all losses resulting from the political event or events in question. To this end, I distinguish between explicit events and ongoing change. The nature of explicit events is that they arrive intermittently at discrete intervals and that they generate an actual loss. Explicit events can be represented by a Poisson jump process. Ongoing change takes the form of continuous activity such as macroeconomic management and monetary policy, legislation, or social and political evolution that affects some or all aspects of the FDI's overall environment and can be represented by geometric Brownian motion.

I find that the China factor effect is almost six times higher on Hong Kong than on Taiwan. For Hong Kong, the China factor raises the political risk level by $329 \%$ in the worst case scenario and by $149 \%$ in the best case. For Taiwan the figures are $60 \%$ and $24 \%$ respectively. Nevertheless, even in the worst case scenario, Hong Kong political risk is considerably lower than China's, which confirms the intuition that Hong Kong could be a lower risk back door avenue to the potentially lucrative Chinese market. Incorporating the cost of political risk into the capital budgeting process as a portfolio consisting of a long position in the FDI and a short position in the insurance policy, I show that otherwise acceptable projects in Hong Kong can become unacceptable when the China factor is taken into consideration.

\section{References}

Bell, M. W., Khor, H.E. \& Kochhar K. [1993], China at the Threshold of a Market Economy, Occasional Paper No. 107, Washington DC: The International Monetary Fund.

Clark, E. A. [1991], Cross Border Investment Risk, London: Euromoney Publications, Plc.. 
Gwartney, J., Lawson, R. \& Block, W. [1996], Economic Freedom of the World: 1975-1995, Vancouver: Fraser Institute.

Khan, M. S., \& Reinhart, C. M. [1995], Capital Flows in the APEC Region, Occasional Paper No. 122, Washington DC: The International Monetary Fund.

Kobrin, S. J. [1979], "Political Risk: A Review and Reconsideration," Journal of International Business Studies 10; pp. 67-80.

Roddock, D. [1986], Assessing Corporate Political Risk, Totowa, N.Y.: Rowman and Littlefield Publishers.

Shapiro, A. C. [1978], "Capital Budgeting for the Multinational Corporation,” Financial Management 7; pp. 7-16.

Stonehill, A. \& Nathanson, L. [1968], "Capital Budgeting and the Multinational Corporation,” California Management Review 4; pp. 39-54.

Tseng, W., Khor, H.E., Kochhar, K., Mihaljek, D. \& Burton, D. [1994], Economic Reform in China: A New Phase, Occasional Paper No. 114, Washington DC: The International Monetary Fund. 